\title{
Vascular Flora of a Riparian Site on the Canadian River, Cleveland County, Oklahoma
}

\author{
Lacy Burgess \\ Department of Geography \\ University of Oklahoma \\ Norman, OK 73019
}

\author{
Bruce W. Hoagland \\ Oklahoma Biological Survey \\ and Department of Geography \\ University of Oklahoma \\ Norman, OK 73019 \\ e-mail: bhoagland@ou.edu
}

This article reports the results of an inventory of the vascular plants from a riparian site in central Oklahoma. One hundred and sixty-three species of vascular plants in 131 genera and 45 families were collected. The most species were collected from the families Asteraceae (32) and Poaceae (26). Fifty-eight species were annuals, 97 perennials, and 8 biennials. Eight species of woody plants were present. Twenty-nine species, or $18 \%$ of the flora, were exotic to Oklahoma. No species listed as threatened or endangered by the U.S. Fish and Wildlife Service nor those tracked by the Oklahoma Natural Heritage Inventory were encountered.

\section{INTRODUCTION}

Although North America has a long history of botanical inventory, exotic species are often under-represented in herbarium collections. Herbarium collections typically document the native flora of an area. Many botanists have historically shown little interest in collecting "weeds" or non-native species. Thus it is difficult to determine the arrival and establishment of exotic and invasive species (Cronk and Fuller, 2001). The same is true of highly disturbed areas which have historically been under-collected by botanists due to higher concentrations of non-native species (Planty-Tabacchi et al., 1996). In recent years such habitats have become of interest, particularly in Europe, because they harbor a greater occurrence and abundance of exotic species (Ferreira and Moreira, 1995; Kowarik, 1995).

The objective of this study is to inventory the flora of a riparian area that has been heavily impacted by natural and anthropogenic disturbances on the Canadian River. Natural disturbances consist of flooding and channel migration, which often destroy extant vegetation. Anthropogenic disturbances at the site include the abandoned City of Norman Municipal Landfill (NML) and sand and gravel removal for an adjacent asphalt plant. The NML now serves as a national test site for United States Geological Survey (USGS) studies of water pollution associated with landfills. The plant species list resulting from this project will serve as a baseline for USGS researchers and their cooperators working at the NML.

\section{STUDY AREA}

The study area occupies 254 hectares on the Canadian River in Cleveland County, Oklahoma $\left(35^{\circ} 16^{\prime} \mathrm{N}, 95^{\circ} 44^{\prime} \mathrm{W}\right)$. The site has been heavily disturbed by a variety of land uses (Figure), including the NML, which was 
active from 1922 until 1985 (Curtis and Whitney, 2003). Active sand excavation occurs in the southern portion of the study area. In addition, the Canadian River is used for recreational purposes by the citizens of Norman. Past agricultural uses include hay fields and livestock grazing.

The dynamics of the Canadian River has also affected plant species composition. The Canadian River is a braided stream that often ceases to flow during summer months. Discharge on the Canadian River averages $310.16 \mathrm{~m}^{3} /$ second (Tortorelli, 1999). A significant flood event in 1987 pushed the river channel approximately $500 \mathrm{~m}$ south to the present location. Other major floods were recorded in 1941, 1948, and 1986 (Curtis and Whitney, 2003).

Climate of the study area is semi-arid continental. The hottest month is July, with an average temperature of $27.8^{\circ} \mathrm{C}$, and the coldest is January, with an average temperature $2.4^{\circ} \mathrm{C}$ (Oklahoma Climate Survey 2006). The growing season is six months long (Bourlier et al., 1987). Topography at the site consists of gently rolling, southwest sloping plains. The soils are Quaternary alluvium on terrace deposits. Two soil types are present, the Gracemore silty clay loam and the Gracemore loamy fine sand and/or clay loams, both of the Gracemore-Gracemont Association (Bourlier et al., 1987). Both soils are frequently flooded, moderately alkaline, and calcareous. Underlying the alluvium is a low permeability unit of shale and mudstone (Eganhouse, et al., 1999).

\section{MATERIALS AND METHODS}

Voucher specimens were collected from $1 \mathrm{~m}^{2}$ plots placed at $23 \mathrm{~m}$ intervals along a transect and from random sites throughout the study area. Collections were made at twoweek intervals from April through November 2005. Vouchers for species exotic to North
America were made from naturalized populations only, thus excluding cultivated and ornamental plants. Specimens were processed at the Robert Bebb Herbarium of the University of Oklahoma (OKL) following standard procedures. Manuals used for specimen identification included Waterfall (1969) and Diggs et al. (1999). Origin, either native or introduced, was determined by using USDA-NRCS (2006). Nomenclature follows the US Department of Agriculture-Natural Resources Conservation Service (USDANRCS, 2006). Voucher specimens were deposited at OKL.

\section{RESULT AND DISCUSSION}

One hundred sixty-three species of vascular plants in 45 families, and 131 genera were collected (Appendix, Table). The Asteraceae (32), Poaceae (26), Fabaceae (11), and Cyperaceae (10), had the greatest number of species. Fifty-eight species of annuals, eight biennials, and 97 perennials were collected. Eight species of woody plants were collected, five of which were primarily represented by young saplings. No federally listed threatened or endangered species, or species tracked by the Oklahoma Natural Heritage Inventory, were encountered during this study. Carduus nutans (Asteraceae) and Rhynchospora nivea (Cyperaceae) were records for Cleveland County (Hoagland et al., 2006).

Twenty-nine species $(18 \%)$ from 14 families were introduced. The family with the greatest number of introduced species was Poaceae (11). Sixteen of the introduced species were annuals or biennial and 13 were perennials. The percentage of exotic species at this site exceeds those reported in recent floristic studies in Oklahoma, which range from 9-13 percent of the flora (Hoagland and Johnson, 2001, 2004a, 2004b; Hoagland and Buthod, 2003, 2004; Hoagland and Wallick, 2003; Hoagland, et al., 2004; Hoagland, et al., 
2004). Given the small size of the flora, exotic species richness would be expected to have a disproportional effect. This is likely augmented by the disturbance history of the site.

Two habitat types predominated at the study site. Wetland and aquatic vegetation included the Canadian River channel and small ephemeral wetlands. Common species included Amorpha fruticosa, Andropogon glomeratus, Eleocharis obtusa, Juncus torreyi, Salix exigua, S. nigra, Schoenoplectus americanus, Tamarix gallica, and Typha latifolia. Disturbed areas and old-fields were sites heavily impacted by the human activities described above. These areas were generally represented by fewer species, many of which were introduced. Common species in this habitat included Ambrosia trifida, Amphiachyris dracunculoides, Convolvulus arvensis, Cyperus esculentus, Bromus tectorum, Helianthus maximiliani, Torilis arvensis, and Verbesina encelioides.

\section{ACKNOWLEDGMENTS}

We thank Jennifer Larsen and Christy Batterson for field assistance.

\section{REFERENCES}

Bourlier, B.G, G.A. Sample, B.G. Swafford, and G. Douthit. 1987. Soil Survey of Cleveland County, Oklahoma. United States Department of Agriculture, Soil Conservation Service.

Cronk, Q.C.B., and J.L.Fuller. 2001. Plant invaders: The threat to natural ecosystems. Earthscan Publications Ltd. Sterling, Virginia, USA.

Curtis J.A. and J.W. Whitney. 2003. Geomorphic and Hydrologic Assessment of Erosion Hazards at the Norman Municipal Landfill, Canadian River Floodplain, Central Oklahoma.
Environment and Engineering

Geoscience. 9:241-257

Diggs, G.M, BL.Lipscomb, and R.J.

O'Kennon. 1999. Shinners and

Mahler's Illustrated Flora of North

Central Texas. Botanical Research

Institute of Texas, Fort Worth, Texas.

Eganhouse, R.P., I.M. Cozzarelli, M.A. Scholl, and L.L. Matthews. 1999. Evidence for Natural Attenuation of Volatile Compounds in the Leachate Plume of a Municipal Landfill Near Norman, Oklahoma. In US Geological Survey Toxic Substances hydrology ProgramProceedings of the Technical Meeting Charleston, South Carolina March 8-

12, 1999. Water-Resources Investigations Report 99-4018C.

Ferreira , M.T. and I.S. Moreira. 1995. The Invasive Component of a River Flora Under the Influence of Mediterranean Agricultural Systems. In Plant Invasions-General Aspects and Special Problems. Editors P. Pysek,K. Prach, M. Rejmanek, and M. Wade. SPB Academic Publishing, Amsterdam, The Netherlands. p. 85103.

Hoagland, B.W. and A.K. Buthod. 2003. Vascular flora of the Keystone Wildlife Management Area, Creek, Pawnee, and Osage Counties, Oklahoma. Oklahoma Native Plant Record. 3:23-37.

Hoagland, B.W. and A.K. Buthod. 2004. Vascular flora of Hugo Lake Wildlife Management Area, Choctaw County, Oklahoma. Southeast Naturalist. 3:701-714.

Hoagland, B.W. and F.L. Johnson. 2001. Vascular flora of the Chickasaw National Recreation Area, Murray County, Oklahoma. Castanea 66:383400. 
Hoagland, B.W. and F.L. Johnson. 2004a. Vascular flora of Chouteau Wildlife Management Area, Wagoner County, Oklahoma. Oklahoma Native Plant Record. 4:30-39.

Hoagland, B.W. and F.L. Johnson. 2004b. Vascular flora of Love Valley Wildlife Management Area, Love County, Oklahoma. Proceedings of the Oklahoma Academy of Science. 83:47-62.

Hoagland, B.W. and F.L. Johnson. 2004c. Vascular flora of Red Slough and Grassy Slough Wildlife Management Areas, Gulf Coastal Plain, McCurtain County, Oklahoma. Castanea. 69:284-296.

Hoagland, B.W. and K. Wallick. 2003. Vascular flora of Oologah Wildlife Management Area in Nowata County, Oklahoma. Proceedings of the Oklahoma Academy of Science. 83:47-62.

Hoagland, B.W., A.K. Buthod, and W. Elisens. 2004. Vascular flora of Washita Battlefield National Historic Site, Roger Mills County, Oklahoma. Sida. 21:1187-1197.

Hoagland, B.W., P. Crawford-Callahan, P. Crawford, and F.L. Johnson. 2004. Vascular flora of Hackberry Flat, Frederick Lake, and Suttle Creek, Tillman County, Oklahoma. Sida. 21:429-445.

Hoagland, B.W., A.K. Buthod, I. Butler, P. Callahan-Crawford, W. Elisens, A. Udasi and R. Tyrl. 2006. Oklahoma Vascular Plants Database. [online]. Available: http://www.biosurvey.ou.edu. (Accessed on 1 March 2006).

Kowarik, I. 1995. On the Role of Alien Species in Urban Flora and
Vegetation. In Plant Invasions-General Aspects and Specia Problems. Editors P. Pysek, K. Prach, M. Rejmanek, and M. Wade. SPB Academic Publishing, Amsterdam, The Netherlands. p. 85103.

Oklahoma Climatological Survey. 2006. Oklahoma climatological data [online]. Available from http://www.ocs.ou.edu/. (Accessed on 1 February 2006).

Palmer, M.W., G.L. Wade, and P. Neal. 1995. Standards for the writing of floras. Bioscience 45:339-345.

Planty-Tabacchi, A.M., E. Tabacchi, R.J. Naiman, C. Deferrari, and H. Decamps. 1996. Invasibility of Species Rich Communities in Riparian Zones. Conservation Biology 10: 598-607.

Pysek, P. and K. Prach. 1993. Plant Invasions and the Role of Riparian Habitats: A Comparison of Four Species Alien to Central Europe. Journal of Biogeography 20: 413-420.

Pysek, P. 1995. On the Terminology Used in Plant Invasion Studies. In Plant Invasions-General Aspects and Special Problems. Editors P. Pysek, K. Prach, M. Rejmanek, and M. Wade. SPB Academic Publishing, Amsterdam, The Netherlands. p. 7181.

Tortorelli, R. L. 1999. Statistical Summaries of Streamflow in Oklahoma Through 1999.

http://pubs.usgs.gov/wri/wri024025/ (accessed 5-27-2006)

USDA, NRCS. 2006. The PLANTS Database (http://plants.usda.gov, 28 June 2006). National Plant Data Center, Baton Rouge, LA 70874-4490 USA.

Waterfall, U.T. 1969. Keys to the Flora of Oklahoma. Published by the author. 
Table Summary of floristic collections made in Cleveland County, Oklahoma.*

\begin{tabular}{|c|c|c|c|c|c|}
\hline Taxonomic grouf & & Species & Native spp. & & Exotic spp. \\
\hline Equisetophyta & & 2 & 2 & & 0 \\
\hline Coniferophyta & & 1 & 1 & & 0 \\
\hline \multirow[t]{3}{*}{ Magnoliophyta } & & 160 & 131 & & 29 \\
\hline & Magnoliopsida & 116 & & 100 & 1 \\
\hline & Liliopsida & 44 & & 31 & 1 \\
\hline Total & & 163 & 134 & & 29 \\
\hline
\end{tabular}

* Table format follows Palmer et al. (1995). 


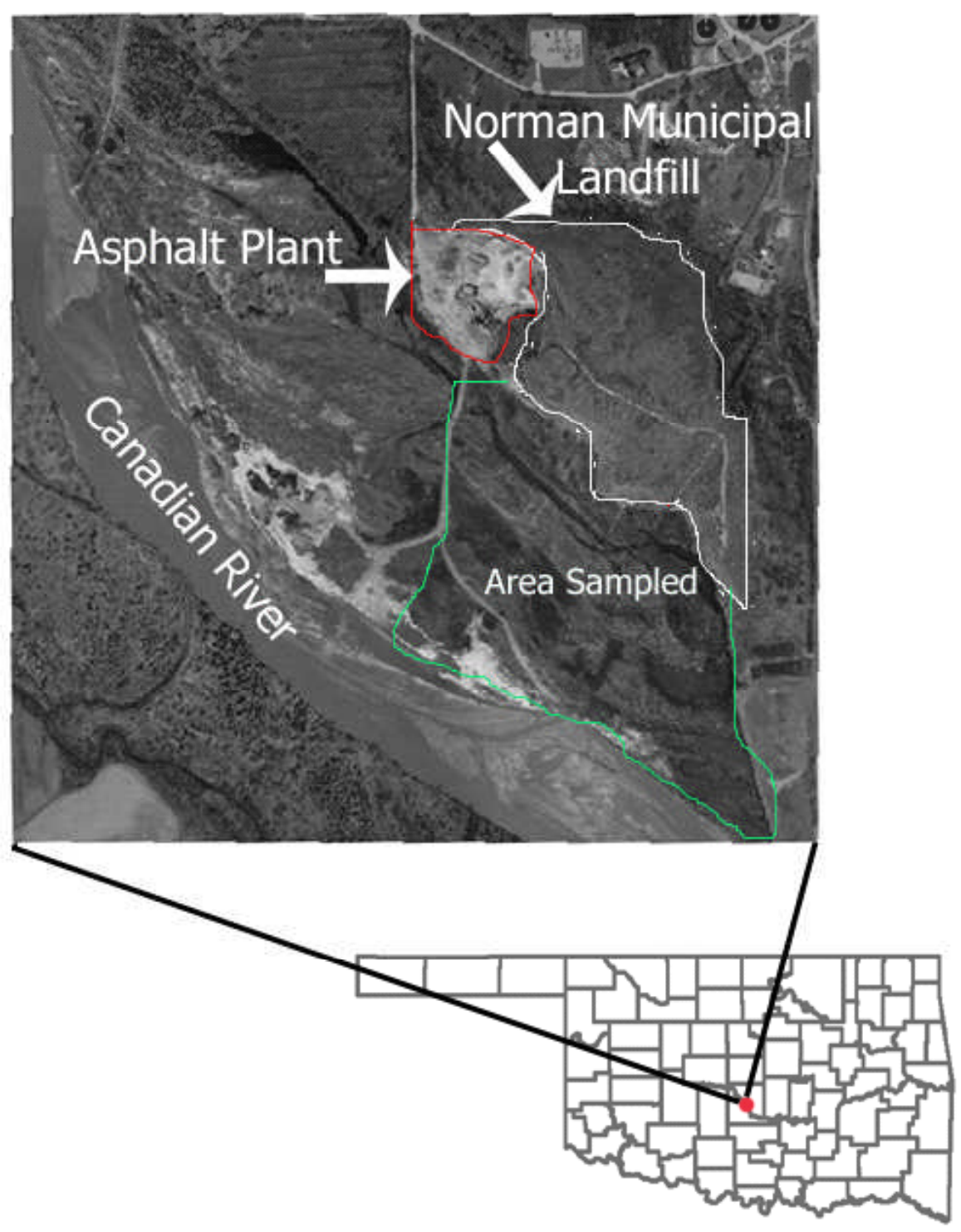

Figure Location of Norman Landfill Site, Cleveland County, Oklahoma. 


\section{APPENDIX}

Annotated species list for a riparian site on the Canadian River in Cleveland County, Oklahoma. The first entry indicates life history $(\mathrm{A}=$ Annual, $\mathrm{B}=$ Biennial, $\mathrm{P}=$ Perennial $)$, followed by habitat (WETL $=$ wetland and aquatic vegetation, DAOF $=$ disturbed areas and old-field vegetation), and collection number. Exotic species are denoted with an asterisk. Voucher specimens were deposited at the Robert Bebb Herbarium at the University of Oklahoma (OKL).

Coniferophyta

Cupressaceae

Juniperus virginiana L. (eastern red cedar): $\mathrm{P}$, DAOF, \#152

\section{Equisetophyta}

\section{Equisetaceae}

Equisetum byemale L. var. affine (Engelm) A.A.

Eat. (scouringrush horsetail): P, WETL, \#15

Equisetum laevigatum A. Braun (smooth

horsetail): P, WETL, \#16

\section{Magnoliophyta \\ Magnoliopsida}

\section{Anacardiaceae}

Rhus glabra L. (smooth sumac): P, DAOF, \#149

Toxicodendron radicans (L.) Kuntze (poison ivy): P, DAOF, not collected\#

\section{Apiaceae}

Cicuta maculata L. (spotted water hemlock): B, WETL, \#150

Torilis arvensis (Huds.) Link* (hedge parsley): A, DAOF, \#85

\section{Asteraceae}

Achillea millefolium L.* (common yarrow): $\mathrm{P}$, DAOF, \#50

Ambrosia psilostachya D.C. (western ragweed):

P, DAOF, \#86

Ambrosia trifida L. (giant ragweed): A, DAOF, Lost\#

Amphiachyris dracunculoides (DC.) Nutt. (prairie broomweed): A, DAOF, \#131

Aphanostephus skirrhobasis DC. (lazy daisy): A, DAOF, \#51
Carduus nutans L.* (nodding plumeless thistle): B, DAOF, \#154

Chrysopsis pilosa Nutt. (soft goldenaster): A, DAOF, \#1

Conyza canadensis (L.) Cronq. (horseweed): A, DAOF, \#132

Coreopsis tinctoria Nutt. (plains coreopsis): P, DAOF, \#133

Croptilon divaricatum (Nutt.) Raf. (slender scratchdaisy): A, DAOF, \#134

Dracopis amplexicaulis (Vahl) Cass. (clasping coneflower): A, DAOF, \#158

Eclipta prostrata (L.) L. (false daisy): P, WETL, \#53

Erigeron annuus (L.) Pers. (daisy fleabane): A, DAOF, \#90

Erigeron philadelphicus L. (Philadelphia fleabane): P, DAOF, \#2

Eupatorium serotinum Michx. (lateflowering thoroughwort): P, DAOF, \#135

Euthamia gymnospermoides Greene (Texas goldentop): P, WETL, \#195

Helianthus hirsutus Raf. (hairy sunflower): P, DAOF, \#138

Helianthus maximiliani Schrad. (Maximilian sunflower): P, DAOF, \#139

Helianthus petiolaris Nutt. (prairie sunflower): A, DAOF, \#4

Heterotheca canescens (DC.) Shinners. (hoary false goldenaster): P, DAOF, \#116

Heterotheca subaxillaris (Lam.) Britt. \& Rusby (camphorweed): A, DAOF, \#56

Iva annua L. (annual marshelder): A, DAOF, \#141

Lactuca canadensis L. (Canada lettuce): B, DAOF, \#5 
Pluchea odorata L. Cass (purple camphorweed): P, DAOF, \#91

Rudbeckia birta L. (blackeyed susan): P, DAOF, \#57

Rudbeckia grandiflora (D. Don) J.F. Gmel. Ex D.C. (blackeyed susan): P, DAOF, \#142

Solidago gigantea Ait. (giant goldenrod): P, DAOF, \#143

Sonchus asper (L.) Hill *(spiny sowthistle): A, DAOF, \#158

Symphyotrichum ericoides (L.) Nesom var. ericoides (white heath aster): P, DAOF, \#196

Thelesperma filifolium (Hook.) Gray var. filifolium (stiff greenthread): P, DAOF, \#145

Verbesina encelioides (Cav.) Benth. \& Hook. f. ex

Gray (golden crownbeard): A, DAOF, \#199

Xanthium strumarium L. (rough cocklebur): A,

DAOF, \#146

Amaranthaceae

Amaranthus cruentuss L. (slim amaranth): A,

DAOF, \#44

\section{Apiaceae}

Cicuta maculata L. (spotted water hemlock): B, WETL, \#117

Conium maculatum L.* (poison hemlock): B, WETL, \#45

Torilis nodosa (L.) Gaetrn.* (knotted

hedgeparsley): A, DAOF, \#148

\section{Apocynaceae}

Apocynum cannabinum L. (Indianhemp): P, WETL, \#47

\section{Asclepiadaceae}

Asclepias viridis Walt. (green antelopehorn): P, $\mathrm{DAOF}, \# 49$

Asclepias arenaria Torr. (sand milkweed): P, DAOF, \#118

\section{Bignoniaceae}

Campsis radicans (L.) Seem. ex. Bureau (trumpet creeper): P, DAOF, \#52

Catalpa bignonioides Walt. (southern catalpa): P, WETL/DAOF, \#6

\section{Boraginaceae}

Heliotropium convolvulaceum (Nutt.) Gray (phlox heliotrope): A, DAOF, \#119

\section{Brassicaceae}

Lepidium virginicum L. (Virginia pepperweed): A, WETL, \#60

Rorippa sessiliflora (Nutt.) A.S. Hitchc. (stalkless yellowcress): A, DAOF/WETL, \#7

Rorippa palustris (L.) Bess. (bog yellowcress): A, WETL, \#120

Campanulaceae

Triodanis perfoliata (L.) Nieuwl. (clasping Venus' looking-glass): A, DAOF, \#8

\section{Caryophyllaceae}

Arenaria serpyllifolia L.* (thymeleaf sandwort): A, DAOF, \#10

\section{Chenopodiaceae}

Chenopodium ambrosioides L.* (Mexican tea): A, WETL, \#9

\section{Convolvulaceae}

Convolvulus arvensis L.* (field bindweed): P, DAOF, \#11

\section{Euphorbiaceae}

Chamaesyce missurica (Raf.) Shinners (prairie sandmat): A, DAOF, \#156

Cnidoscolus texanus (Muell.-Arg) Small (bull nettle): P, DAOF, \#157

Euphorbia marginata Pursh (snow on the mountain): A, DAOF, \#159

\section{Fabaceae}

Amorpha fruticosa L. (false indigo): P, WETL, \# 95

Chamaecrista fasciculata (Michx) Greene var. fasciculata, (partridge pea): P, DAOF, \#96

Desmanthus illinoensis (Michx.) MacM. ex. B.L. Robins \& Fern. (Illinois bundleflower): P, DAOF, \#97

Desmodium ciliare (Muhl ex Willd.) DC. (hairy small-leaf ticktrefoil): P, DAOF, \#191 
Indigofera miniata Ortega (coastal indigo): $\mathrm{P}$, DAOF/WETL, \#123

Lathyrus hirsutus L.* (Caley pea): A, DAOF, \# 66

Lespedera stuevei $\mathrm{Nutt}$.(tall lespedeza): P, DAOF, \#192

Medicago lupulina L. (black medick): P, DAOF, \#193

Melilotus officialis (L.) Lam. (yellow sweet clover): B, DAOF, \#99

Strophostyles helvola (L.) Elliott (trailing fuzzybean): A, WETL./DAOF, \#160 Vicia villosa Roth* (winter vetch): B, DAOF, \#162

\section{Gentianaceae}

Eustoma exaltatum (L.) Salisb. ex G. Don ssp. russellianum (Hook.) Kartesz

(showy prairie gentian): P, DAOF, \#144

Sabatia campestris Nutt. (prairie rose): A, DAOF/WETL, \#100

\section{Lamiaceae}

Lamium amplexicaule L.* (henbit deadnettle):

A, DAOF, \#20

Lycopus americanus Muhl. ex W. Bart.

(American water horehound): P, WETL, \#163

Teucrium canadense L. (American germander): P, DAOF, \#165

\section{Lythraceae}

Ammannia coccinea Rottb. (valley redstem): A, WETL, \#87

Lythrum alatum Pursh (winged lythrum): P, DAOF/WETL, \#126

Rotala ramosior (L.) Koehne (lowland rotala): A, WETL, \#70

\section{Malvaceae}

Callirhoe involucrata (Torr. \& Gray) Gray

(purple poppymallow): P, DAOF, \#22 Mirabilis nyctaginea (Michx.) MacM. (heartleaf four o'clock): P, DAOF, \#101

\section{Onagraceae}

Gaura coccinea Nutt. ex Pursh (scarlet beeblossom): P, DAOF, \#23

Gaura mollis James (velvetweed): P, DAOF, \#24

Gaura villosa Torr. (woolly beeblossom): P, DAOF/WETL, \#127

Ludwigia peploides (Kunth) Raven (floating primrose-willow): P, WETL, \#193

Oenothera laciniata Hill (cutleaf eveningprimrose): A, DAOF, \#37

Oenothera biennis L. (common eveningprimrose): B, DAOF/WETL, \#84

\section{Oxalidaceae}

Oxalis stricta L. (common yellow oxalis): P, DAOF, \# 72

\section{Papaveraceae}

Argemone polyanthemos (Fedde) G.B. Ownbey (crested pricklypoppy): A, DAOF, \#161

\section{Plantaginaceae}

Plantago patagonica Jacq. (woolly plantain): A, DAOF, \#25

\section{Polygonaceae}

Polygonum hydropiperoides Michx. (swamp smartweed): P, WETL, \#75

Polygonum lapathifolium L. (curlytop knotweed): A, WETL, \#194

Polygonum ramosissimum Michx. (bushy knotweed): A, WETL, \#102

Rumex crispus L.* (curly dock): P, WETL, \#76

\section{Primulaceae}

Samolus valerandi L. ssp. parviflorus (Raf.) Hulten (seaside brookweed): P, WETL, \#78

\section{Ranunculaceae}

Clematis terniflora DC.* (sweet autumn virginsbower): P, DAOF, \#79

Ranunculus sceleratus L. var. sceleratus (cursed buttercup): P, WETL, \#80

\section{Rubiaceae}

Galium aparine L. (stickywilly): A, DAOF, \#129 
Sherardia arvensis L.* (blue fieldmadder): A, DAOF, \#39

\section{Salicaceae}

Populus deltoides Bart. ex Marsh. (eastern cottonwood): P, WETL, \#175

Salix exigua Nutt. (sandbar willow): P, WETL, \#176

Salix nigra Marsh (black willow): P, WETL, \#177

\section{Scrophulariaceae}

Agalinis heterophylla (Nutt.) Small ex. Britt. (prairie false foxglove): A, DAOF/WETL, \#178

Agalinis aspera (Dougl. ex. Benth.) Britt. (tall false foxglove): A, DAOF/WETL, \#179

Castilleja indivisa Engelm.(Indian paintbrush): A, DAOF, \#111

Leucospora multifida (Michx.) Nutt. (narrowleaf paleseed): A, DAOF, \#88

Veronica anagallis-aquatica $\mathrm{L}$. (water speedwell): P, WETL, \#40

Veronica peregrina L. (neckweed): A, WETL, \#180

\section{Solananceae}

Datura wrightii Regel (sacred thorn-apple): P, DAOF, \#182

Physalis angulata L. (cutleaf ground cherry): A, WETL/DAOF, \#183

Physalis heterophylla Nees (clammy groundcherry): P, DAOF, \#184

Solanum elaeagnifolium Cav. (silverleaf nightshade): P, WETL, \#185

Solanum physalifolium Rusby (hoe nightshade): A, DAOF, \#186

Solanum rostratum Dunal (buffalobur nightshade): A, DAOF, \#187

\section{Tamariaceae}

Tamarix gallica L.* (salt cedar): P, WETL/DAOF, \#114

\section{Valerianaceae}

Valerianella radiata (L.) Dufr. (beaked cornsalad): A, DAOF, \#42

\section{Verbenaceae}

Phyla lanceolata (Michx.) Greene (lanceleaf frogfruit): P, WETL, \#83

Phyla nodiflora (L.) Greene (turkey tangle frogfruit): P, WETL, \#82

Verbena stricta Vent. (hoary verbena): P, DAOF, \#189

\section{Vitaceae}

Vitis cinerea (Engelm.).Engelm ex. Millard (sweet grape): P, DAOF, \#190

\section{Liliopsida}

Commelinaceae

Commelina virginica L. (Virginia dayflower): P, DAOF, \#151

\section{Cyperaceae}

Carex festucacea Schkuhr ex Willd. (fescue sedge): P, WETL, \#62

Cyperus esculentus L.* (yellownutsedge): P, WETL, \#12

Cyperus odoratus L. (fragrant flatsedge): A, WETL, \#153

Cyperus squarrosus L. (bearded flatsedge): A, WETL, \#54

Cyperus strigosus L. (strawcolored flatsedge): P, WETL, \#174

Eleocharis obtusa (Willd.) J.A. Schultes (blunt spikerush): P, WETL, \#13

Fuirena simplex Vahl (western umbrella-sedge): P, WETL, \#14

Schoenoplectus americanus (Pers.) Volk. ex. Schinz \& Keller (chairmaker's bulrush): P, WETL, \#155

Schoenoplectus maritimus (L.) Lye (cosmopolitan bulrush): P, WETL, \#122

Rhynchospora nivea Boeckl. (showy whitetop): P, WETL, \#112 


\section{Iridaceae}

Sisyrinchium angustifolium P. Mill. (narrowleaf blue-eyed grass): P, DAOF, \#18

\section{Juncaceae}

Juncus acuminatus Michx. (tapertip rush): P, WETL, \#67

Juncus biflorus Ell. (bog rush): P, WETL, \#195

Juncus marginatus Rostk. (grassleaf rush): $\mathrm{P}$, WETL, \#124

Juncus torreyi Coville (Torrey's rush): P, WETL, \#19

\section{Lilaceae}

Ornithogalum umbellatum L.* (star of bethleham): P, DAOF, \#21

\section{Poaceae}

Andropogon ternarius Michx. (splitbeard bluestem): P, DAOF, \#170

Andropogon glomeratus (Walt.) B.S.P. (bushy

bluestem): P, WETL, \#164

Aristida oligantha Michx. (prairie threeawn) : A, DAOF, \# 174

Bromus catharticus Vahl* (rescuegrass) : A, DAOF, \# 89

Bromus japonicus Thunb. ex Murr.* (Japanese brome): A, DAOF, \#26

Bromus tectorum L.* (cheatgrass): A, DAOF, \#27

Cenchrus spinifex Cav. (coastal sandbur): A, DAOF/WETL, \#166

Cynodon dactylon (L.) Pers.* (Bermuda grass): P, DAOF, \#105

Dichanthelium acuminatum (Sw.) Gould \& C.A. Clark var. fasciculatum (Torr.)

Freckmann (western panicgrass): P, DAOF, \#174

Digitaria cognata (J.A. Schultes) Pilger (fall witchgrass): P, DAOF, \#125
Echinochloa crus-galli (L.) Beauv.*

(barnyardgrass): A, DAOF/WETL, \#28

Elymus canadensis L. (Canada wildrye): $\mathrm{P}$, DAOF, \#168

Elymus virginicus L. (Virginia wildrye): P, DAOF, \#29

Festuca brevipila Tracey* (hard fescue): P, DAOF, \#169

Hordeum pusillum Nutt. (little barley): A, WETL/DAOF, \#30

Koeleria macrantha (Ledeb.) J.A. Schultes (prairie Junegrass): P, DAOF/ WETL, \#31

Leptochloa fusca (L.) Kunth ssp. fascicularis (Lam. ) Snow (bearded sprangletop): P, DAOF/WETL, \#32

Lolium perenne L. ssp. multiflorum (Lam.) Husnot* (Italian ryegrass): P, DAOF, \#33

Panicum virgatum $\mathrm{L}$. (switchgrass): $\mathrm{P}$, WETL/DAOF, \#35

Phragmites australis (Cav.) Trin. ex. Steudel* (common reed): P, WETL, \#171

Polypogon monspeliensis (L.) Desf.* (rabbitsfoot grass): A, WETL, \#36

Saccharum ravennae (L.) L.* (Ravenna grass): P, WETL, \#172

Schizachyrium scoparium (Michx.) Nash var. scoparium (little bluestem): P, DAOF, \#173

Setaria parviflora (Poir.) Kerguèlen (marsh bristlegrass): P, WETL, \#109

Sorghum halepense (L.) Pers.* (Johnson grass): P, DAOF, \#110

Sporobolus cryptandrus (Torr.) Gray (sand dropseed): P, DAOF, \#128

\section{Typhaceae}

Typha domingensis Pers. (southern cattail)): P, WETL, \#188 